\title{
Spatial Distribution of Metals in the ICM: Evolution of the Iron Excess in Relaxed Galaxy Clusters
}

\author{
Ang Liu,${ }^{1,2,3 \star}$ Paolo Tozzi,${ }^{1} \dagger$ Heng $\mathrm{Yu}^{4}$, Sabrina De Grandi ${ }^{5}$, Stefano Ettori ${ }^{6,7}$ \\ ${ }^{1}$ INAF - Osservatorio Astrofisico di Arcetri, Largo E. Fermi, I-50122 Firenze, Italy \\ ${ }^{2}$ Department of Physics, Sapienza University of Rome, I-00185 Rome, Italy \\ ${ }^{3}$ Department of Physics, University of Rome Tor Vergata, I-00133, Rome, Italy \\ ${ }^{4}$ Department of Astronomy, Beijing Normal University, Beijing, China 100875 \\ ${ }^{5}$ INAF - Osservatorio Astronomico di Brera, Via E. Bianchi, 46, I-23807 Merate (LC), Italy \\ ${ }^{6}$ INAF - Osservatorio di Astrofisica e Scienza dello Spazio, via Pietro Gobetti 93/3, 40129 Bologna, Italy \\ ${ }^{7}$ INFN, Sezione di Bologna, viale Berti Pichat 6/2, I-40127 Bologna, Italy
}

Accepted XXX. Received YYY; in original form ZZZ

\begin{abstract}
We investigate the spatial distribution of iron in the intra-cluster medium in a selected sample of 41 relaxed clusters in the redshift range $0.05<z<1.03$ using Chandra archival data. We compute the azimuthally-averaged, deprojected $Z_{\mathrm{Fe}}$ profile of each cluster out to $\sim 0.4 r_{500}$, and identify a peak in the distribution of iron followed by a flatter distribution at larger radii. Due to the steep gradient both in gas density and abundance, we find that the emission-weighted iron abundance within $0.2 r_{500}$, which entirely includes the iron peak in most of the cases, is on average $\sim 25 \%$ higher than the mass-weighted value, showing that spatially resolved analysis and accurate deprojection are key to study the evolution of iron enrichment in the very central regions of cool core clusters. We quantify the extent of the iron distribution in each cluster with a normalized scale parameter $r_{\mathrm{Fe}}$, defined as the radius where the iron abundance excess is half of its peak value. We find that $r_{\mathrm{Fe}}$ increases by a factor of $\sim 3$ from $z \sim 1$ to $z \sim 0.1$, suggesting that the spatial distribution of iron in the ICM extends with time, possibly due to the mixing with the mechanical-mode feedback from the central galaxy. We also find that the iron mass excess within $0.3 r_{500}$, when normalized to the total baryonic mass within the same region, does not evolve significantly, showing that this iron mass component is already established at $z \sim 1$.
\end{abstract}

Key words: galaxies: clusters: intracluster medium, X-rays: galaxies: clusters

\section{INTRODUCTION}

Clusters of galaxies are the largest gravitationally-bound systems in the universe. Growing through gravitational instability from the fluctuations in the primordial density field (see Kravtsov \& Borgani 2012, and references therein), not only can their abundance be used to trace the cosmic evolution thanks to a strong dependence on the cosmological parameters (Allen et al. 2011; Mantz et al. 2016), but they can also be considered approximately as closed boxes which retain the imprints of the evolution of the member galaxies and the surrounding intracluster medium (ICM, see, e.g., Böhringer \& Werner 2010). The X-ray emitting ICM is by far the dominant baryonic component across the full range of virial masses of galaxy groups and clusters (Lin et al. 2012),



$\dagger$ E-mail: ptozzi@arcetri.astro.it (P. Tozzi) and is constituted of a hot, diffuse, optically-thin plasma in collisional equilibrium, enriched by heavy elements produced mainly by supernovae (SNe) explosions. The abundance of heavy elements can be directly measured through the equivalent width of the corresponding emission lines in the X-ray energy range, thanks to the assumption of collisional equilibrium and the low optical depth of the ICM. Self-absorption of the most prominent resonant lines has been shown to be an important diagnostic in high $\mathrm{S} / \mathrm{N}$ X-ray spectra, but it has noticeable effect on the line emission only in the highdensity central regions, as recently measured in the Hitomi observation of the innermost $\sim 30 \mathrm{kpc}$ of the Perseus cluster for the $K_{\alpha}$ line of He-like iron (Hitomi Collaboration et al. 2017). Despite the simple framework outlined here, the relative abundance of the different heavy elements, the cosmic evolution of their abundance and of their spatial distribution are shaped by complex physics which are not fully understood yet. 
In the most massive, hence most luminous clusters, given the high temperatures reached in the ICM $(k T>3$ $\mathrm{keV}$ ), most of the heavy elements are highly or fully ionized. The most prominent feature from heavy elements is the $K_{\alpha}$ emission line complex from He-like and H-like iron at $6.7-6.9 \mathrm{keV}$ rest-frame. The detection of other elements is much easier at lower temperatures $(k T<3 \mathrm{keV})$ and low redshifts, and typically requires high $\mathrm{S} / \mathrm{N}$ spectra (see De Grandi \& Molendi 2009; Tamura et al. 2009; Sanders \& Fabian 2011; Mernier et al. 2017). For these reasons, iron is the only heavy element that can be detected in galaxy clusters up to $z \sim 1.6$ (Rosati et al. 2009; Tozzi et al. 2013, 2015), with some tentative detection at $z \sim 2$ (see Mantz et al. 2017, where iron emission is detected at $2.6 \sigma$ confidence level). Therefore, several studies in the last ten years focused on the cosmic evolution of the global iron abundance. Previous studies of evolution in the metallicity of the ICM were consistent with a significant evolution of a factor of $\sim 2$ in the range $0<z<1.3$, at least in Chandra data (e.g. Balestra et al. 2007; Maughan et al. 2008; Anderson et al. 2009), although more recent results are consistent with little or no evolution (Ettori et al. 2015; McDonald et al. 2016).

The spatial distribution of heavy elements, typically azimuthally averaged at each radius, became an important aspect to be taken into account in evolutionary studies, given that the relative abundance can strongly vary with radius. The strongest gradients are observed in the cool cores, defined as the central cluster regions where the gas cooling time is shorter than a given reference value (for an extensive discussion of the definition of cool core see Hudson et al. 2010). Ideally, one would aim at resolving the chemical evolution as a function of the radius. Recently, Mantz et al. (2017) found negative evolution with redshift in the intermediate regions $\left(0.1 r_{500}<r<0.5 r_{500}\right)$, while at smaller $\left(r<0.1 r_{500}\right)$ and larger radii $\left(r>0.5 r_{500}\right)$, the ICM is consistent with a constant iron abundance at least up to $z \sim 1.2$. On the other hand, systematic investigations of the spatially resolved evolution of iron abundance in the ICM with XMM-Newton data support the general trend of a negative evolution with redshift in the central regions of cool-core clusters, and an almost constant behavior at radii larger than $0.4 r_{500}$ (Ettori et al. 2015). Therefore, we can conclude that studies dominated by X-ray selected clusters, as those previously mentioned, agree on a general early $(z>1.5)$ enrichment, and on the presence of some moderate evolution in the iron abundance, but do not agree on the radial range where this evolution is taking place. In addition, the same presence of evolution of ICM enrichment in the cluster population has been recently challenged by studies based on an SPT-SZ selected sample at $z<1.5$, where no significant evolution has been found (McDonald et al. 2016). This result may be at least partially reconciled with the previous results on the basis of a much lower fraction of cool cores, which are more enriched in metals, among SZ- selected clusters (Rossetti et al. 2017). However, this hypothesis still needs to be tested across a combined cluster sample free of any selection bias.

To summarize, there are significant differences in several relevant aspects among recent studies, and we are far from having a coherent description of the cosmic evolution of iron in the ICM. Not only we do not know which is the radial range where most of the iron evolution takes place, but we do not even know whether some amount of evolution does actually take place, nor whether it depends on the cluster selection itself. A general lesson from the literature, is that it is mandatory to follow the spatial distribution of the iron abundance as a function of redshift, halo mass, and thermodynamical properties of the ICM to successfully constrain a physical model of its chemical enrichment.

This goal can be barely achieved on the basis of presentday data and X-ray facilities. Currently, due to the limited statistics of high- $z$ cluster samples, the photon-starved Xray follow-up observation of high- $z$ clusters, and the frustrating perspective of X-ray astronomy (with Chandra, the only high-resolution instrument, rapidly losing efficiency in the soft band), it is a hard task to improve the measurement of the evolution of the iron abundance in the ICM. Despite this, the cosmic evolution of heavy element enrichment of the ICM across the cosmic epochs is gaining increasing interest. In particular, the relative ratio of the abundance of various ions provides important clues on the ratio of type Ia supernovae (SNIa) and core-collapse supernovae (SNcc), which eject different amount of heavy elements (Werner et al. 2008; Madau \& Dickinson 2014; Maoz \& Graur 2017). Thus the evolution of ICM enrichment can be used, in principle, to constrain the $\mathrm{SNe}$ rates in cluster galaxies, once absolute yields are robustly constrained. In turn, the prediction of absolute yields constitutes a key aspect which is still highly debated (Finoguenov et al. 2000; Böhringer et al. 2004; De Grandi \& Molendi 2009; Matsushita et al. 2013), but is far beyond the goal of this paper.

For these reasons we adopt an approach that begins by exploiting nearby and bright clusters, where we can successfully constrain the distribution of the heavy elements as a function of the cluster radius, and eventually extend our investigation to higher-z targets. The general properties of the iron distribution at low redshifts are well known. De Grandi \& Molendi (2001) and De Grandi et al. (2004) investigated the projected iron profiles for a sample of 17 low- $z$ clusters observed by BeppoSAX, and clearly showed that non-cool-core clusters had flat iron profiles while cool-core clusters show a strong iron enhancement towards the center. This property is now commonly observed in all the regular/relaxed clusters, suggesting a physical link between the processes that shape the thermodynamics of the ICM and its chemical enrichment. In particular, in high $\mathrm{S} / \mathrm{N}$ data, it is possible to identify a well defined peak in the iron distribution above the average abundance level, which allows one to measure a relative excess of iron with respect to the global iron distribution. This excess may be associated with relatively recent star formation events in the brightest cluster galaxy (BCG, see De Grandi et al. 2004), but its origin and evolution are still unclear. The shape of the iron excess is clearly very sensitive to the many complex physical processes occurring in the center of galaxy clusters, such as gas motions driven by outflows of the central AGN (Sijacki et al. 2007; Roediger et al. 2007; Fabian 2012), the sloshing of cool cores (Markevitch \& Vikhlinin 2007; Ghizzardi et al. 2014), stochastic gas motions (Rebusco et al. 2005), sinking of highly enriched low-entropy gas (Cora 2006; Cora et al. 2008), and galactic winds (Tornatore et al. 2004; Romeo et al. 2006). In De Grandi et al. (2014), our group has already shown that, at least in the case of WARPJ1415.1+3612 (the brightest cool-core cluster at $z \sim 1$ ), the peak in the iron 
distribution is significantly narrower than in local clusters, when compared to the stellar light distribution of the underlying BCG.

We argue that in this respect, the exquisite angular resolution of Chandra may play a key role in providing well defined abundance profiles not only at low redshift. Therefore, in this work, we start a systematic investigation of the spatial distribution of iron abundance in the ICM at different epochs (up to $z \sim 1$ ) with a limited but ideal sample of relaxed clusters observed with Chandra. Massive, relaxed clusters constitute the best targets where we can attempt to disentangle different components in the iron distribution. Our goal here is to extend the few works currently available in the literature (Baldi et al. 2012; Ettori et al. 2015; Mantz et al. 2017), putting most of the emphasis on the spatial distribution and its physical implications. Our final aim is to build a universal physical model for the iron distribution and use it to extend our study to all the clusters with X-ray detections, irrespective of their $\mathrm{S} / \mathrm{N}$.

The paper is organized as follows. In Section 2, we describe the sample selection, the dataset extracted from the Chandra archive, and the data reduction. In Section 3, we briefly discuss the global properties of the sample. In Section 4 , we present the deprojected spatially-resolved analysis and derive the deprojected $Z_{\mathrm{Fe}}$ profiles. In Section 5, we discuss the correlation between pseudo entropy, gas cooling time and iron abundance. In Section 6, we discuss our results on the central iron excess and the width of the iron peak. Our conclusions are presented in Section 7 . Throughout this paper, we adopt the seven-year WMAP cosmology $\left(\Omega_{\Lambda}=0.73\right.$, $\Omega_{m}=0.27$, and $H_{0}=70.4 \mathrm{~km} \mathrm{~s}^{-1} \mathrm{Mpc}^{-1}$ (Komatsu et al. 2011). Quoted error bars correspond to a $1 \sigma$ confidence level unless noted otherwise.

\section{SAMPLE SELECTION AND DATA REDUCTION}

To achieve our science goal, the selection criteria include both the physical properties of the targets and the data quality. In this work we do not aim at measuring the global evolution of iron as a function of cosmic epoch, and we use an optimally selected cluster sample to fully exploit the power of spatially resolved spectroscopy.

First, we set our requirements on the physics of the targets. To constrain the shape of the iron distribution in the ICM as a function of the radial distance from the center, we are required to select clusters for which an azimuthally averaged $Z_{\mathrm{Fe}}$ value as a function of the cluster radius is well defined, which implies an approximate spherical symmetry and a relaxed dynamical state. Obviously, any major merger event makes the temperature and abundance distribution highly asymmetric and patchy, undermining any attempt to define a meaningful radial distribution of $Z_{\mathrm{Fe}}$. Therefore, this requirement forces us to select relaxed, round-shaped clusters classified as such on the basis of morphological information.

We start from the sample presented in Mantz et al. $(2015)$, where the symmetry $(s)$-peakiness $(p)$-alignment $(a)$ (SPA) criterion is used to select relaxed clusters. By applying the criterion $s>0.87, p>-0.82$, and $a>1.00$ to a sample of 361 clusters, they identify 57 clusters in the red- shift range $0.01<z<1.03$ as relaxed (see Mantz et al. 2015, for details).

We check the $0.5-7 \mathrm{keV}$ Chandra images of these clusters, obtained by merging all the useful observations. We exclude from the sample the clusters which show clear signatures of non-equilibrium previously missed by the SPA test, such as obvious substructures in X-ray surface brightness distribution (e.g., A133 and RXJ1347.5-1145). There are 52 clusters surviving these criteria. We also include a relaxed cluster which passes the SPA criterion but is not included in Mantz et al. (2015): PLCKG266.6-27.3 at $\mathrm{z}=0.940$, a remarkable cluster with a high $\mathrm{S} / \mathrm{N}$ and high redshift (see Bartalucci et al. 2017).

We require a number of net counts $\geq 6000$ in the $0.5-$ $7 \mathrm{keV}$ energy band and within the extraction radius of $\sim 0.4 r_{500}$ to have at least 6 independent annuli with more than 1000 net counts each. This threshold is required to achieve typical errors on the iron abundance of the order of $30 \%$ or less in each ring (see Yu et al. 2011). We also require that $\sim 0.4 r_{500}$ (our maximum extraction radius) be included entirely within the ACIS field of view. Depending on the position of the aimpoint, the maximum radius from the cluster center covered by ACIS is about 8 arcmin, which corresponds to $\sim 400 \mathrm{kpc}$ at $z \sim 0.04$ in our seven-year WMAP cosmology. If we assume a typical $r_{500}$ of $1 \mathrm{Mpc}$, this threshold thus excludes some nearby clusters (e.g. Perseus). There are 41 clusters left after applying the data quality threshold. Our final sample of the 41 relaxed clusters in the redshift range $0.05<z<1.03$ is listed in Table 1, where we show the redshift and position of X-ray emission peak of each cluster, and the details of the Chandra data we used in this work. The X-ray emission peak is determined as the position of the brightest pixel of the point source extracted image in 0.5-7 keV band, smoothed with a Gaussian function with $\mathrm{FWHM}=1.5^{\prime \prime}$.

We performed a standard data reduction starting from the level=1 event files, using the CIAO 4.9 software package, with the most recent version (at the time) of the Chandra Calibration Database (CALDB 4.7.8). When observations are taken in the VFAINT mode, we run the task acis_process_events to flag background events that are most likely associated with cosmic rays and remove them. With this procedure, the ACIS particle background can be significantly reduced compared to the standard grade selection. The data are filtered to include only the standard event grades $0,2,3,4$ and 6 . We checked visually for hot columns left after the standard reduction. For exposures taken in VFAINT mode (the large majority of our dataset), there are practically no hot columns or flickering pixels left after filtering out bad events. We also apply CTI correction to ACIS-I data. We finally filter time intervals with high background by performing a $3 \sigma$ clipping of the background level using the script analyze_ltcrv. The final effective exposure times are generally very close to the original observing time.

When the concentric annuli for spectral analysis are selected, we extract the full spectrum after masking unresolved sources, which are previously identified with wavdetect and eventually checked manually to identify faint sources missed by the detection algorithm due to the dominating ICM emission. For clusters with multiple observations, we extract the spectrum and compute the response matrix file and ancillary response file for each Obsid separately, and fit the spectra 
Table 1. The sample and data we used in this work. The coordinate is the position of the peak of X-ray emission.

\begin{tabular}{|c|c|c|c|c|c|}
\hline Cluster & $z_{\mathrm{opt}}$ & RA(J2000) & $\operatorname{Dec}(J 2000)$ & ObsID/Detector(ACIS-I/-S) & Exptime (ks) \\
\hline Hydra-A & 0.055 & 09:18:05.7 & $-12: 05: 43.9$ & $575 \mathrm{I}$ & 20.9 \\
\hline Abell2029 & 0.077 & $15: 10: 56.1$ & $+05: 44: 41.3$ & 891S,4977S,6101I & 106.7 \\
\hline Abell2597 & 0.083 & $23: 25: 19.8$ & $-12: 07: 26.7$ & $6934 \mathrm{~S}, 7329 \mathrm{~S}$ & 110.2 \\
\hline Abell478 & 0.088 & $04: 13: 25.1$ & $+10: 27: 57.2$ & $1669 \mathrm{~S}, 6102 \mathrm{I}$ & 51.5 \\
\hline PKS0745-191 & 0.103 & $07: 47: 31.3$ & $-19: 17: 39.2$ & $12881 \mathrm{~S}$ & 117.8 \\
\hline RXJ1524.2-3154 & 0.103 & $15: 24: 12.9$ & $-31: 54: 22.5$ & 9401S & 40.8 \\
\hline Abell1068 & 0.138 & $10: 40: 44.5$ & $+39: 57: 11.5$ & $1652 \mathrm{~S}$ & 26.8 \\
\hline Abell2204 & 0.152 & $16: 32: 46.9$ & $+05: 34: 32.0$ & 499S,6104I,7940I & 96.6 \\
\hline Abell1204 & 0.171 & $11: 13: 20.5$ & $+17: 35: 40.9$ & $2205 \mathrm{I}$ & 23.6 \\
\hline Abell383 & 0.188 & 02:48:03.4 & $-03: 31: 46.1$ & $524 \mathrm{I}, 2320 \mathrm{I}, 2321 \mathrm{~S}$ & 48.7 \\
\hline RXJ0439.0+0520 & 0.208 & 04:39:02.2 & $+05: 20: 43.6$ & 527I,9369I,9761I & 37.9 \\
\hline ZwCL2701 & 0.214 & $09: 52: 49.1$ & $+51: 53: 06.0$ & $12903 \mathrm{~S}$ & 95.6 \\
\hline RXJ1504.1-0248 & 0.215 & $15: 04: 07.5$ & $-02: 48: 16.8$ & 4935I, 5793I & 52.3 \\
\hline ZwCL2089 & 0.235 & 09:00:36.8 & $+20: 53: 39.9$ & $10463 \mathrm{~S}$ & 40.5 \\
\hline RXJ2129.6+0005 & 0.235 & $21: 29: 39.9$ & $+00: 05: 21.0$ & $552 \mathrm{I}, 9370 \mathrm{I}$ & 39.4 \\
\hline RXJ1459.4-1811 & 0.236 & $14: 59: 28.8$ & $-18: 10: 45.2$ & $9428 \mathrm{~S}$ & 39.3 \\
\hline Abell1835 & 0.253 & 14:01:01.9 & $+02: 52: 44.2$ & 6880I,6881I,7370I,495S,496S & 221.5 \\
\hline Abell3444 & 0.253 & $10: 23: 50.2$ & $-27: 15: 23.1$ & $9400 \mathrm{~S}$ & 36.3 \\
\hline MS1455.0+2232 & 0.258 & $14: 57: 15.1$ & $+22: 20: 34.3$ & 7709I,543I,4192I & 107.8 \\
\hline MS2137.3-2353 & 0.313 & $21: 40: 15.2$ & $-23: 39: 40.2$ & $928 \mathrm{~S}, 4974 \mathrm{~S}, 5250 \mathrm{~S}$ & 119.5 \\
\hline MACSJ2229.7-2755 & 0.324 & $22: 29: 45.2$ & $-27: 55: 36.7$ & $3286 \mathrm{~S}, 9374 \mathrm{~S}$ & 30.3 \\
\hline MACSJ0947.2+7623 & 0.345 & $09: 47: 12.7$ & $+76: 23: 13.9$ & $7902 \mathrm{~S}$ & 38.3 \\
\hline MACSJ1931.8-2634 & 0.352 & $19: 31: 49.6$ & $-26: 34: 33.8$ & $9382 \mathrm{I}$ & 97.6 \\
\hline MACSJ1115.8+0129 & 0.355 & $11: 15: 51.9$ & $+01: 29: 55.9$ & $3275 \mathrm{I}, 9375 \mathrm{I}$ & 53.1 \\
\hline RXJ1532.9+3021 & 0.362 & $15: 32: 53.8$ & $+30: 20: 59.3$ & 14009S & 88.2 \\
\hline MACSJ0011.7-1523 & 0.378 & $00: 11: 42.9$ & $-15: 23: 21.2$ & 3261I,6105I & 58.8 \\
\hline MACSJ1720.2+3536 & 0.391 & $17: 20: 16.8$ & $+35: 36: 25.5$ & 3280I,6107I,7718I & 60.8 \\
\hline MACSJ0429.6-0253 & 0.399 & 04:29:36.1 & $-02: 53: 08.4$ & $3271 \mathrm{I}$ & 22.4 \\
\hline MACSJ0159.8-0849 & 0.404 & 01:59:49.3 & $-08: 49: 58.2$ & 3265I,6106I,9376I & 72.5 \\
\hline MACSJ2046.0-3430 & 0.423 & $20: 46: 00.5$ & $-34: 30: 18.2$ & $5816 \mathrm{I}, 9377 \mathrm{I}$ & 49.2 \\
\hline IRAS09104+4109 & 0.442 & $09: 13: 45.5$ & $+40: 56: 28.6$ & $10445 \mathrm{I}$ & 75.9 \\
\hline MACSJ0329.6-0211 & 0.450 & 03:29:41.6 & $-02: 11: 46.8$ & 3257I,3582I,6108I,7719I & 76.1 \\
\hline MACSJ1621.3+3810 & 0.463 & $16: 21: 24.8$ & $+38: 10: 08.8$ & $\begin{array}{l}{[3254 \mathrm{I}, 3594 \mathrm{I}, 6109 \mathrm{I}, 6172 \mathrm{I},} \\
7720 \mathrm{I}, 9379 \mathrm{I}, 10785 \mathrm{I}]\end{array}$ & 161.1 \\
\hline $3 \mathrm{C} 295$ & 0.464 & $14: 11: 20.5$ & $+52: 12: 09.9$ & $2254 \mathrm{I}$ & 87.2 \\
\hline MACSJ1423.8+2404 & 0.543 & $14: 23: 47.9$ & $+24: 04: 42.6$ & $4195 \mathrm{~S}$ & 38.9 \\
\hline SPT-CLJ2331-5051 & 0.576 & $23: 31: 51.1$ & $-50: 51: 54.0$ & 9333I,11738I & 31.8 \\
\hline SPT-CLJ2344-4242 & 0.596 & $23: 44: 44.0$ & $-42: 43: 12.4$ & 13401I,16135I,16545I & 118.0 \\
\hline SPT-CLJ0000-5748 & 0.702 & 00:01:00.0 & $-57: 48: 33.1$ & 9335I & 28.4 \\
\hline SPT-CLJ2043-5035 & 0.723 & $20: 43: 17.6$ & $-50: 35: 32.2$ & $\begin{array}{l}\text { 13478I } \\
{[14017 \mathrm{I}, 14018 \mathrm{I}, 14349 \mathrm{I}, 14350 \mathrm{I}}\end{array}$ & 73.3 \\
\hline PLCKG 266.6 & 0.940 & $06: 15: 51.8$ & $-57: 46: 47.3$ & $\begin{array}{l}\text { 14351I,14437I,15572I,15574I } \\
\text { 15579I,15582I,15588I,15589I] }\end{array}$ & 240.6 \\
\hline CLJ1415+3612 & 1.030 & $14: 15: 11.1$ & $+36: 12: 02.7$ & $12255 \mathrm{~S}, 12256 \mathrm{~S}, 13118 \mathrm{~S}, 13119 \mathrm{~S}$ & 276.5 \\
\hline
\end{tabular}

with linked parameters. Background spectra are extracted from a selection of regions far from the ICM emission in each Obsid. When the ICM emission fills the entire field of view, we use the background generated from the "blank sky" files with the blanksky script in CIAO, which finds the correct blank sky files, reprojects them to match the data, and properly determines the scaling. The "blank sky" background is used only in one case: ObsID 575 for Hydra-A. The spectra fitting is done with Xspec 12.9.0. The apec thermal plasma emission model (Smith et al. 2001) is used to fit the ICM spectrum, with abundance relative to the solar values of Asplund et al. (2009). C-statistics (Cash 1979) are used in the spectra fitting. Galactic absorption is described by the model tbabs (Wilms et al. 2000), where the Galactic column density $\mathrm{NH}_{\mathrm{Gal}}$ is frozen to the value corresponding to the cluster position in the HI survey of Kalberla et al. (2005)

\section{GLOBAL PROPERTIES}

In this section we derive the global properties of each cluster, namely the X-ray redshift, the emission weighted global temperature, and the radius $r_{500}$. The global properties will be used uniquely to characterize the sample, and will not be used in our analysis except for the normalization of the radii to the value of $r_{500}$.

We measure the global temperature from the cumulative spectrum extracted in the region $0.1 r_{500}<r<\sim 0.4 r_{500}$. This choice is often adopted in the literature to obtain temperature values that more closely trace the virial value, 




Figure 1. Histogram of the values of the global temperature $\langle k T\rangle$ measured in the radial range $0.1 r_{500}<r<0.4 r_{500}$ with a singletemperature apec model.

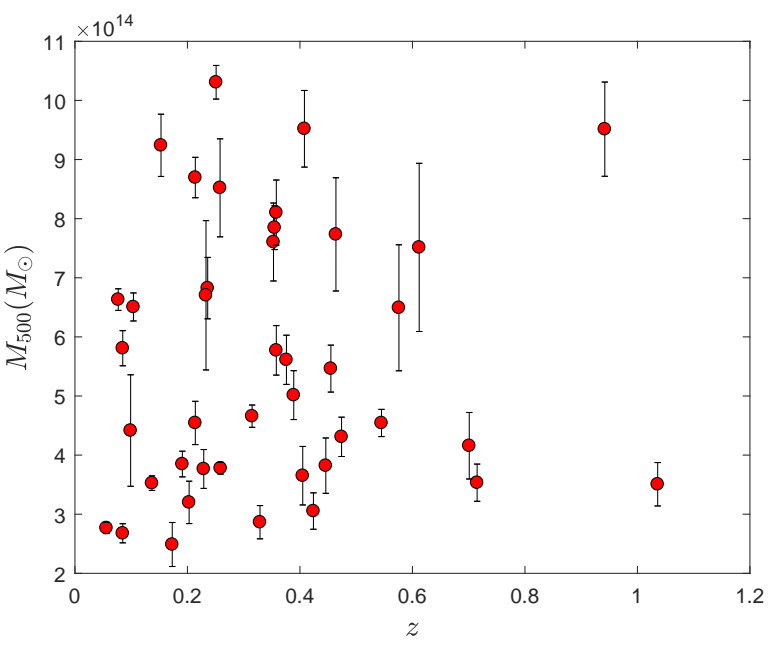

Figure 2. The $M_{500}$ obtained from the scale relation $M_{500} \propto$ $\langle k T\rangle^{3 / 2} / E(z)$ plotted against redshift for our sample.

avoiding the effect of the cool core when present. We use a single-temperature apec model, therefore $\langle k T\rangle$ is an emission weighted value resulting from the range of temperatures present in the explored radial range. To estimate $r_{500}$, we use the average relation described in Vikhlinin (2006):

$r_{500}=\frac{0.792}{h E(z)}\left(\frac{\langle k T\rangle}{5 \mathrm{KeV}}\right)^{0.53} \mathrm{Mpc}$

where $E(z)=\left(\Omega_{\mathrm{m}}(1+z)^{3}+\Omega_{\Lambda}\right)^{0.5}$. The global temperatures $\langle k T\rangle$ and $r_{500}$ are evaluated iteratively until we obtain a stable temperature. As shown in column 3 of Table 2, our sample spans a large range of temperatures of $3<\langle k T\rangle<12$ $\mathrm{keV}$ with a peak at $6 \mathrm{keV}$. As we show in Figure 1, we find higher temperatures at higher redshift, however this does not necessarily imply significantly larger masses. We check the mass $M_{500}$ with the self-similar model $M_{500} \propto\langle k T\rangle^{3 / 2} / E(z)$,

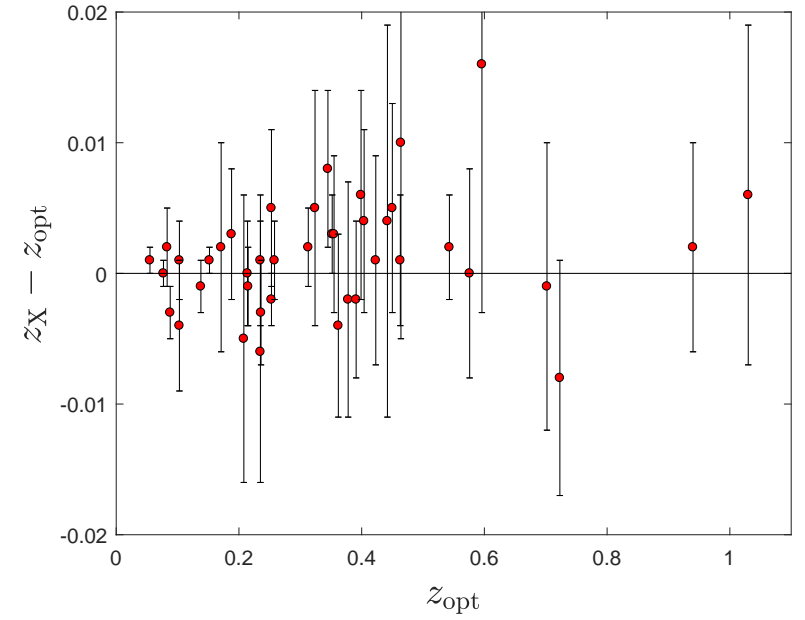

Figure 3. The difference between X-ray redshift and optical redshift of all clusters.

with the normalization measured in Vikhlinin et al. (2006), and plot $M_{500}$ as a function of redshift in figure 2. We notice that the mass range spanned by our sample is not significantly changing with redshift. In particular, the mass range (spanning a factor of $\sim 5$ ) is more or less equally populated up to redshift $z \sim 0.6$, with only four clusters at $z>0.6$.

We note that, in principle, we can measure mass and $r_{500}$ directly from our spectrally resolved analysis, by measuring the total mass from the hydrostatic equation, and computing the radius corresponding to the average overdensity $\Delta=500$ with respect to the critical density at the cluster redshift. However, in order to do this we should measure robust density and temperature profiles and therefore sample the ICM emission carefully at radii larger than $r_{500}$, which is beyond the goal of this paper. We perform a check on the four clusters in our sample with the highest $S / N$ (Abell2597, PKS0745-191, MACSJ2229.7-2755 and MACSJ1423.8+2404) and confirm that the extrapolated value of $r_{500}$ obtained from our hydrostatic mass profile is consistent with that obtained from Equation 1 within 5\%, and that the mass proxy we used in Figure 2 is accurate within $8 \%$.

Since the measurement of iron abundance is sensitive to the X-ray redshift, we also investigate possible discrepancies between the optical redshift and the X-ray redshift, as obtained from the fit to the global emission with an apec model leaving the redshift parameter free. For simplicity, we measure the X-ray redshift of the clusters by fitting the 2.0$7.0 \mathrm{keV}$ spectra so that the best-fit redshift is determined uniquely by the H-like and He-like iron line complex at $6.7-$ $6.9 \mathrm{keV}$ rest frame. As shown in Figure 3, we find that the $\mathrm{X}$-ray and spectroscopic redshifts are consistent within $\sim 1 \sigma$. We fix the redshift at the best-fit global X-ray value in the following X-ray analysis. 


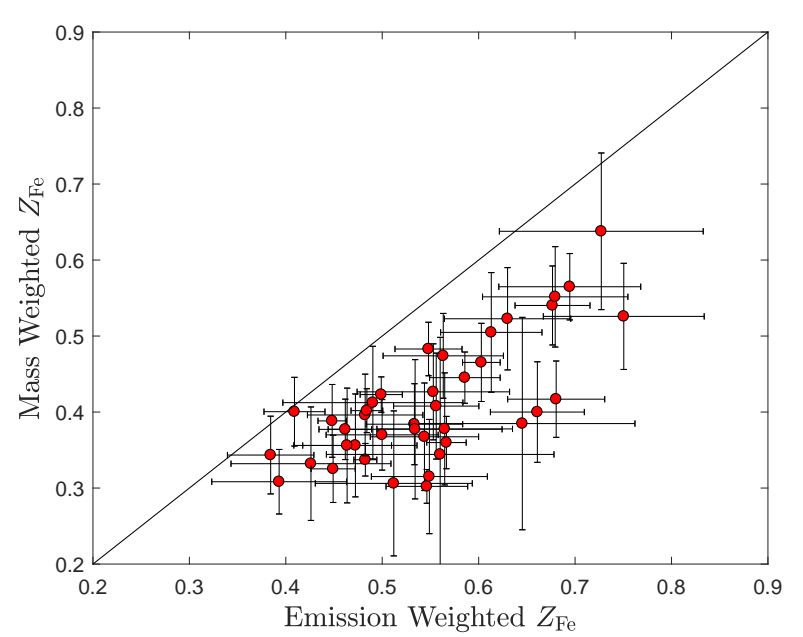

Figure 4. The global iron abundance $\left\langle Z_{\mathrm{Fe}}\right\rangle \times M_{\text {gas }}$ versus emission weighted iron abundance obtained with our spatially resolved analysis in the radial range $r<0.2 r_{500}$.

\section{MEASUREMENT OF THE IRON DENSITY PROFILE}

To measure the iron density profiles (together with the temperature and density profiles), the first step is to derive the the azimuthally-averaged, projected $Z_{\mathrm{Fe}}$ profile, as measured in a series of concentric annuli centered in the peak of X-ray emission out to the maximum radius $\sim 0.4 r_{500}$. The annuli are chosen with an adaptive criterion based on a smoothly varying $\mathrm{S} / \mathrm{N}$ threshold on the $0.5-7 \mathrm{keV}$ image, to ensure a roughly equal number of net counts $\geq 1000(0.5-7 \mathrm{keV}$ band $)$ in each bin for the inner annuli, and a doubled number of net counts in the outermost ring. This choice is key to keep a comparable quality of the spectral fit in the outer regions, which are mostly affected by the background due to the rapidly declining ICM density profile and the correspondingly larger extraction regions. The number of independent bins per cluster ranges from 6 to 13 .

The next step is the measurement of the actual 3D profile from the projected one, under the assumption of spherical symmetry. Both the projct model in Xspec (Arnaud 1996) and the tool dsdeproj presented in Sanders \& Fabian (2007) can perform a direct and non parametric deprojection of ICM spectra. However, projct may produce large unphysical oscillations in the $3 \mathrm{D}$ profiles in some situations (see Fabian et al. (2006), and examples in Russell et al. (2008)). This instability has been claimed to be due to departure from spherical or ellipsoidal symmetry or the presence of multiphase gas (see, e.g., Fabian et al. 2006). In this work, we use the tool dsdeproj (version 1.2) to produce 3D profiles: it deprojects the spectrum of a shell by subtracting the rescaled count rate of the foreground and background emission.

Our spectral analysis provides profiles for the temperature, iron abundance and the electron density, which can be used to compute the gas density. These three quantities are essentially independent: the electron density mainly depends on the normalization of the emission, with little effects from temperature and abundance. Temperature measurements are determined by spectral shape and by line ratio, and therefore relatively independent from the line intensities. Abundances are measured directly from the equivalent width of the emission lines. Temperature, metallicity and electron density can become strongly coupled in presence of strong gradients across the annulus or along the line of sight, a case in which the spectra from single annuli can no longer be approximated with a single temperature apec model. Clearly, within the limitation of our data, we assume that the angular resolution (number of annuli) in our analysis is sufficient to provide quasi-isothermal spectra.

The deprojection procedure provides us with the iron abundance, $Z_{\mathrm{Fe}}$, temperature and gas density, hence total ICM mass, within each spherical shell. Therefore, we can finally calculate the mass-weighted iron abundance $Z_{\mathrm{mw}}$ defined as $Z_{\mathrm{mw}} \equiv \sum\left(Z_{F e}^{i} \cdot M_{\mathrm{gas}}^{i}\right) / \sum M_{\mathrm{gas}}^{i}$, where $i$ is the index of the shell. We compare $Z_{\mathrm{mw}}$ with the average spectral abundance $\left\langle Z_{\mathrm{Fe}}\right\rangle$ measured from a single-temperature fit of the global emission within the same radius. This is the quantity that is often reported in the literature, mainly because of the difficulty in resolving the ICM profiles for low S/N data (typically medium and high- $z$ clusters). The average abundance can be well approximated with the emission-weighted value $Z_{\text {ew }}$, defined as $\int_{0}^{R} \Lambda(T, Z) n_{e}^{2} Z_{\mathrm{Fe}} / \int_{0}^{R} \Lambda(T, Z) n_{e}^{2}$, where $R$ is the extraction radius. In Figure 4 , we show $Z_{\mathrm{mw}}$ versus $\left\langle Z_{\mathrm{Fe}}\right\rangle$ for all the clusters, computed within $0.2 r_{500}$, which entirely includes the iron peak in most of the cases, and therefore the difference between $Z_{\mathrm{mw}}$ and $\left\langle Z_{\mathrm{Fe}}\right\rangle$ is maximized. This radial range usually is described with at least $4-6$ shells, since it is the region with the brightest emission. We find that the average abundance is about $25 \%$ higher than the mass weighted value. Qualitatively, the result is expected, since $\left\langle Z_{\mathrm{Fe}}\right\rangle \sim Z_{\mathrm{ew}}$ is weighted by $\Lambda(k T, Z) n_{e}^{2}$, and higher $Z_{\mathrm{Fe}}$ is ubiquitously associated with much higher density and slightly lower temperature, therefore corresponds to higher emission weights.

We note that the quantity $Z_{\mathrm{mw}} \times M_{\text {gas }}$ is by construction different from $\left\langle Z_{\mathrm{Fe}}\right\rangle \times M_{\text {gas }}$. The use of $\left\langle Z_{\mathrm{Fe}}\right\rangle \times M_{\text {gas }}$ as a proxy for the mass of iron in the ICM may provide a significant artificial increase of the iron mass. In addition, any cosmic evolution of temperature and density gradients in the core of clusters would impact also on the observed evolution of the average iron abundance. Although this effect is expected to be smaller when including regions beyond $0.2 r_{500}$ (therefore for bright clusters at low and medium redshift), for high- $z$ clusters with strong cool cores we do expect to obtain abundance values $\sim 25 \%$ higher from single measurements based on global emission. The overall impact on iron evolution clearly depends on the quality of the sample and the evolution of the cool-core clusters, and it is beyond the goal of this paper. We claim that the study of the evolution of chemical properties of ICM should be based on mass-weighted quantities in all cases, obtained directly from spatially resolved spectral analysis, when possible, or by physically motivated models of the ICM profiles that allow one to associate massweighted quantities to global quantities. In this way, the uncertainties will be directly associated with the adopted models, allowing a better control on the predictions of iron abundance distributions for the low $\mathrm{S} / \mathrm{N}$ groups and cluster population. 

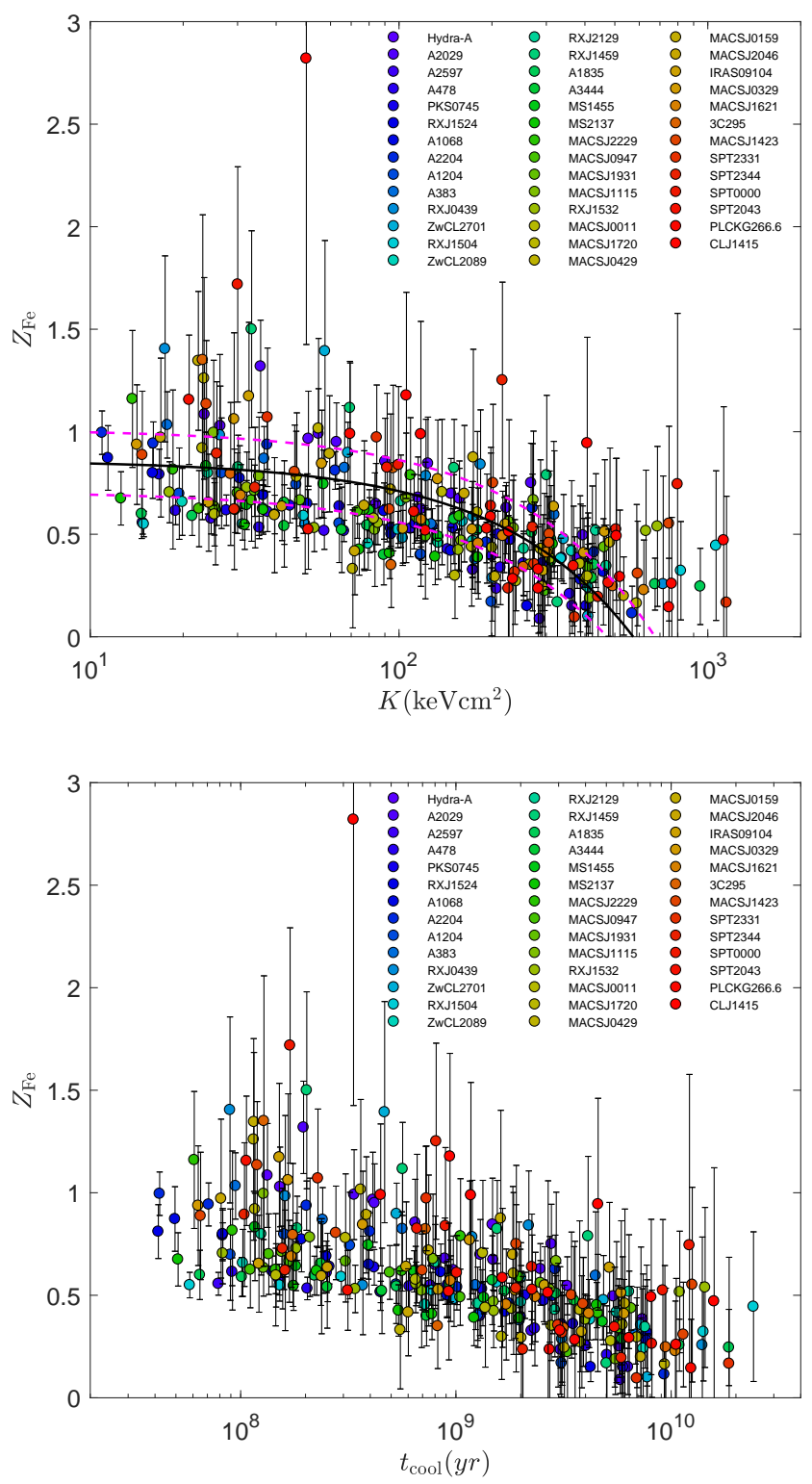

Figure 5. Upper panel: The distribution of $Z_{\mathrm{Fe}}$ versus $K\left(k T, n_{\boldsymbol{e}}\right)$ of all the measured bins in the clusters. The color code denotes the redshift of the clusters, from the lowest (blue) to the highest (red). The solid line is the best fit linear model. The dashed lines show the rms dispersion of the distribution. Lower panel: The distribution of $Z_{\mathrm{Fe}}$ versus the cooling time $t_{\mathrm{cool}}$.

\section{METALLICITY-PSEUDO ENTROPY AND METALLICITY-COOLING TIME RELATIONS}

Given the lack of correlation between density and temperature, the quantity $K\left(k T, n_{e}\right) \equiv k T \cdot n_{e}^{-2 / 3}$ as a function of the radius, often called pseudo-entropy, has been historically used to characterize the thermal history of the ICM (Ponman et al. 1999). Pseudo-entropy stays constant during any adiabatic process, like adiabatic compression, while it may change due to gain or loss of internal energy, mostly due to shocks, turbulence dissipation, or cooling. Therefore, its behavior as a function of radius can be used to identify regions dominated by shock heating (with a slope $\propto r^{1.1}$, see Tozzi \& Norman 2001), adiabatic compression (flat profiles) and cooling. Since the metallicity also has a strong dependence with radius in some clusters, it has been previously claimed that metallicity and pseudo entropy may be associated. In particular, significant increases in metallicity are expected, and often observed, in regions of low entropy where the cooling of the ICM may be associated with events of star formation triggered by the gas dropping out of the cold phase. For example, in local clusters, $Z_{\mathrm{Fe}}$ and $K\left(k T, n_{e}\right)$ are found to have a negative correlation (De Grandi et al. 2004; Leccardi et al. 2010; Ghizzardi et al. 2014).

On these premises, we first investigate the correlation between $Z_{\mathrm{Fe}}$ and $K\left(k T, n_{e}\right)$ for all the independent bins in the clusters of our sample. We fit the $Z_{\mathrm{Fe}}-K\left(k T, n_{e}\right)$ distribution with a linear function $Z=Z_{0}-\alpha \cdot K / 1000$, and obtain the best fit values $Z_{0}=0.86 \pm 0.18$ and $\alpha=1.49 \pm 0.51$. The $Z_{\mathrm{Fe}}{ }^{-}$ $K\left(k T, n_{e}\right)$ distribution and the best fit function are shown in the upper panel of Figure 5.

Similarly, we also show the distribution of $Z_{\mathrm{Fe}}-t_{\mathrm{cool}}$ in the lower panel of Figure 5 . The cooling time of the gas $t_{\text {cool }}$ is defined as the gas enthalpy divided by the energy loss per unit volume (Peterson \& Fabian 2006):

$t_{\mathrm{cool}}=\frac{\frac{5}{2} n_{e} k T}{n_{e}^{2} \Lambda(T, Z)}$,

where $\Lambda(T, Z)$ is the cooling function, which is associated with the energy density emitted by a radiative cooling ICM with a given temperature and metallicity (Boehringer \& Hensler 1989; Sutherland \& Dopita 1993). We find that the $Z_{\mathrm{Fe}}$ shows a similar correlation with $t_{\mathrm{cool}}$ as with $K\left(k T, n_{e}\right)$, given the strong similarity of the two quantities.

The interpretation of this average, highly scattered relation, may be understood only on the basis of a comprehensive model for chemical enrichment of the ICM through the lifetime of groups and clusters. The association of higher abundance values with low entropy and shorter cooling time gas may be, in fact, not simply associated with gas cooling and star formation with consequent local chemical enrichment, but the result of two independent process, like radiative cooling and iron production and diffusion. These processes are both more efficient at the cluster center, but proceed independently and with different time scales. Namely, the spatially resolved analysis we present in this paper is a first step towards a comprehensive model.

\section{MEASURING THE WIDTH OF THE IRON EXCESS PROFILE}

In this section we present the characterization of the distribution of iron abundance throughout the ICM, focusing on the innermost regions. To do that, we fit the deprojected profile of the iron abundance with a phenomenological model with no direct physical meaning. There are two models which have been used in the literature to fit the profiles of ICM iron abundance: a double- $\beta$ model (Santos et al. 2012), and the empirical function provided by Mernier et al. (2017), which is a simple power-law for $r>\sim 0.02 r_{500}$ (in the innermost regions they model the possible decrease of the metallicity by subtracting a Gaussian). However, we often see a strong 


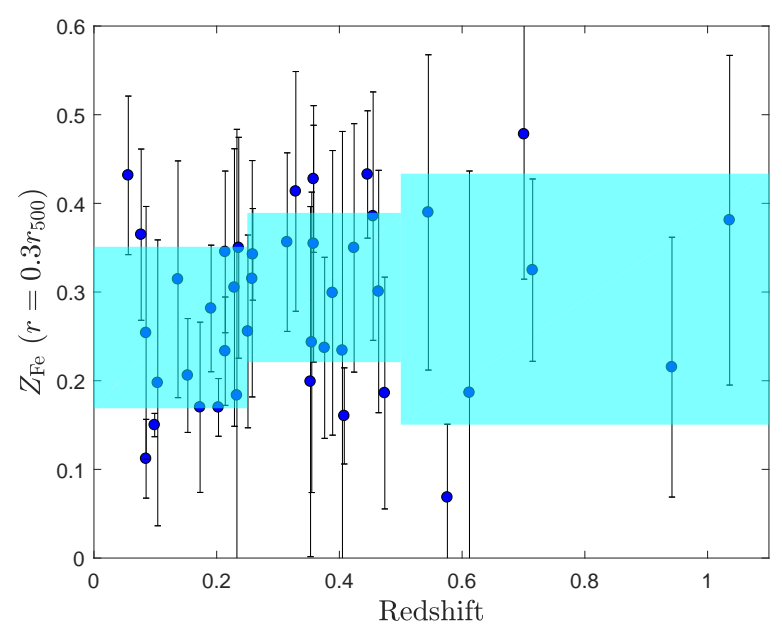

Figure 6. The iron abundance at $r=0.3 r_{500}$ for all the clusters. Shaded area shows the $r m s$ dispersion across three redshift bins: [0.05,0.25], [0.25,0.5], [0.5,1.03].

abundance gradient beyond $\sim 0.02 r_{500}$, and therefore adopt a simpler model which is preferable with respect to a composite model with many free parameters (6 in the case of a double- $\beta$ model). Given the low number of bins we have (particularly for the high- $z$ clusters) we want to have no more than 3 free parameters. We adopt a single $\beta$ model in the form

$Z_{\mathrm{Fe}}(r)=Z_{0} \cdot\left(1+\left(r / r_{0}\right)^{2}\right)^{-\beta}$.

The best fits are obtained minimizing the $\chi^{2}$ over the 3 free parameters. The profiles and the best fit models of all the clusters are presented in the appendix.

After fitting the profiles, we first obtain the iron abundance at $r=0.3 r_{500}$ for all the clusters, and plot $Z\left(0.3 r_{500}\right)$ versus redshift in figure 6 . We group the clusters into three redshift bins: [0.05,0.25], [0.25,0.5], [0.5,1.03], and calculate the average value and $r m s$ dispersion respectively. As a result, we find that $Z\left(0.3 r_{500}\right)$ of the three redshift bins are $0.26,0.30$, and 0.29 , with $\mathrm{rms}$ dispersion of $0.10,0.08$, and 0.13 . This result suggests that the average iron abundance at $0.3 r_{500}$ is consistent with a constant value at $\sim 0.3 Z_{\odot}$ at least up to redshift 1.

The width of the iron profiles is quantified with a newly defined scale parameter $r_{\mathrm{Fe}}$, expressed in units of $r_{500}$. The definition of $r_{\mathrm{Fe}}$ is as follows. We calculate the excess abundance profile $Z_{\text {exc }}(r)=Z(r)-Z\left(0.3 r_{500}\right)$, and the peak excess abundance $Z_{\text {exc, } 0}=Z_{\text {peak }}-Z\left(0.3 r_{500}\right)$, where $Z_{\text {peak }}$ is the peak abundance. Rather than directly adopting $Z_{0}$ of the best-fit beta-model as $Z_{\text {peak }}$, we instead compute the average value within $0.02 r_{500}$ to have a more robust estimate. Finally, we define $r_{\mathrm{Fe}}$ as the radius where $Z_{\mathrm{exc}}\left(r_{\mathrm{Fe}}\right)$ is half of $Z_{\mathrm{exc}, 0}$. The distribution of $r_{\mathrm{Fe}}$ values is listed in Table 2.

Since the distribution of iron differs significantly in coolcore and non-cool-core clusters, the width of iron profiles may also be correlated with the strength of cool-core. To decouple the evolution of the iron distribution from that of the cool core in the clusters, we check whether $r_{\mathrm{Fe}}$ can quantify the broadening of the iron peak, or just shows the strength of the cool core. As a representative parameter, we use the

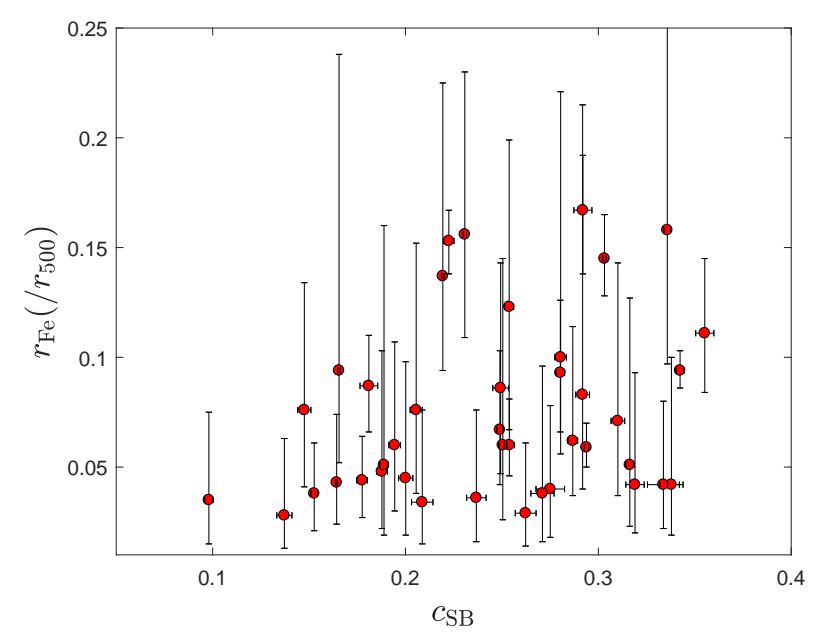

Figure 7. The scale of the iron excess peak, $r_{\mathrm{Fe}}$, plotted versus the cool core strength $c_{\mathrm{SB}}$ (as defined in Santos et al. 2008).

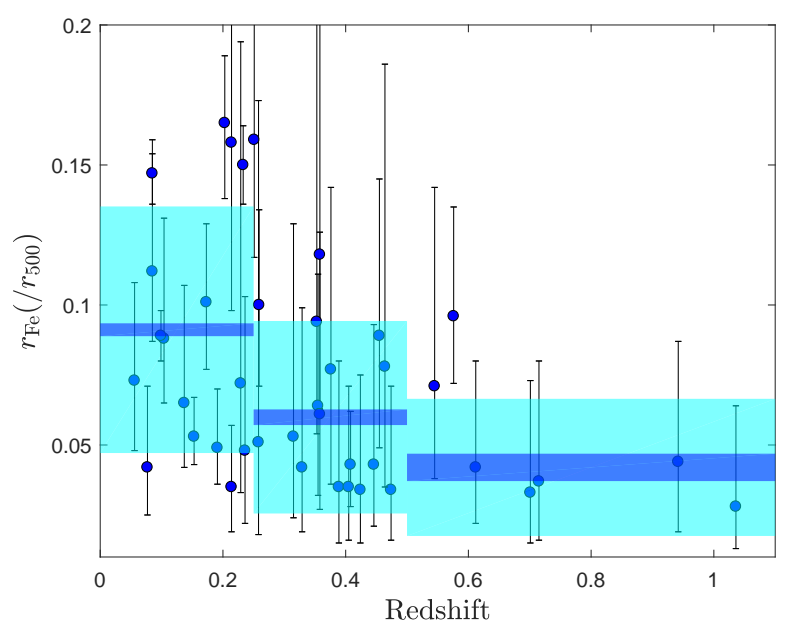

Figure 8. The scale of the iron excess peak, $r_{\mathrm{Fe}}$, plotted versus redshift. Blue and cyan shaded areas show the weighted average and $r m s$ in three redshift bins, respectively.

surface brightness concentration $c_{\mathrm{SB}}$, defined as the ratio of the flux within $40 \mathrm{kpc}$ and $400 \mathrm{kpc}$ (Santos et al. 2008). We plot $r_{\mathrm{Fe}}$ versus $c_{\mathrm{SB}}$ in figure 7 . Spearman's test is performed on the distribution taking into account the uncertainties in $c_{\mathrm{SB}}$ and $r_{\mathrm{Fe}}$, with a resulting $\rho=0.18 \pm 0.11$, corresponding to a very weak correlation with the probability of null hypothesis $p=0.31 \pm 0.26$. This result suggests no significant correlation between $r_{\mathrm{Fe}}$ and $c_{\mathrm{SB}}$, and hence $r_{\mathrm{Fe}}$ can quantify the width of the iron distribution independently from the strength of the cool core.

In figure 8 , we show the scale of the iron excess peak $r_{\mathrm{Fe}}$ as a function of redshift. Since the scatter in $r_{\mathrm{Fe}}$ is very large, we again group the data into three redshift bins $[0.05,0.25]$, $[0.25,0.5]$, and $[0.5,1.03]$. The results show that $r_{\mathrm{Fe}}$ decreases significantly with redshift, despite the large scatter in the low redshift bin. We interpret the significant increase of $r_{\mathrm{Fe}}$ from high- $z$ to low- $z$ as a clear evolution in the spatial dis- 
Table 2. Main results on the clusters. Column 1: cluster name; Column 2: global redshift of the ICM; Column 3: global temperature of the ICM within $0.1 r_{500}-0.4 r_{500}$; Column 4: $r_{500}$ in Mpc measured using the global temperature in column 3; Column 5: the scale of the iron excess peak: $r_{\mathrm{Fe}}$ in $r_{500}$; Column 6: the cool core strength $c_{\mathrm{SB}}$ (as defined in Santos et al. (2008)); Column 7: iron mass excess within $0.3 r_{500}$; Column 8: total gas mass within $0.3 r_{500}$.

\begin{tabular}{|c|c|c|c|c|c|c|c|}
\hline Cluster & X-ray redshift & $k T$ & $r_{500} / \mathrm{Mpc}$ & $r_{\mathrm{Fe}} / r_{500}$ & $c_{\mathrm{SB}}$ & $M_{\mathrm{Fe}}^{\mathrm{exc}} /\left(10^{9} M_{\odot}\right)$ & $M_{\mathrm{gas}} /\left(10^{13} M_{\odot}\right)$ \\
\hline Hydra-A & $0.056_{-0.001}^{+0.001}$ & $3.94_{-0.13}^{+0.14}$ & 1.00 & $0.067_{-0.025}^{+0.036}$ & $0.249 \pm 0.002$ & $0.972 \pm 0.165$ & $0.666 \pm 0.001$ \\
\hline Abell2029 & $0.077_{-0.001}^{+0.001}$ & $6.89_{-0.19}^{+0.13}$ & 1.30 & $0.043_{-0.019}^{+0.023}$ & $0.164 \pm 0.001$ & $2.873 \pm 0.634$ & $2.250 \pm 0.002$ \\
\hline Abell2597 & $0.085_{-0.003}^{+0.001}$ & $3.89^{+0.122}$ & 0.96 & $0.145_{-0.017}^{+0.020}$ & $0.303 \pm 0.001$ & $1.612 \pm 0.592$ & $0.690 \pm 0.001$ \\
\hline Abell478 & $0.085_{-0.002}^{+0.003}$ & $6.35_{-0}^{+0.35}$ & 1.24 & $0.094_{-0.042}^{+0.144}$ & $0.165 \pm 0.001$ & $2.175 \pm 0.923$ & $2.389 \pm 0.003$ \\
\hline PKS0745-191 & $0.104_{-0.002}^{+0.002}$ & $6.86_{-0.25}^{+0.31}$ & 1.28 & $0.137_{-0.043}^{+0.042}$ & $0.219 \pm 0.001$ & $4.772 \pm 3.222$ & $2.338 \pm 0.002$ \\
\hline RXJ1524.2-3154 & $0.099_{-0.003}^{+0.002}$ & $5.36_{-1.014}^{+1.125}$ & 1.12 & $0.094_{-0.008}^{+0.009}$ & $0.342 \pm 0.002$ & $1.825 \pm 0.293$ & $0.825 \pm 0.001$ \\
\hline Abell1068 & $0.137_{-0.001}^{+0.003}$ & $4.70_{-0.17}^{-1.15}$ & 1.03 & $0.062_{-0.025}^{+0.052}$ & $0.287 \pm 0.003$ & $1.030 \pm 0.319$ & $0.839 \pm 0.001$ \\
\hline Abell2204 & $0.153_{-0.001}^{+0.001}$ & $8.69_{-0}^{+0.17}$ & 1.41 & $\begin{array}{l}.059_{-0.009}^{+0.025} \\
0.011\end{array}$ & $0.294 \pm 0.001$ & $4.186 \pm 0.973$ & $2.596 \pm 0.002$ \\
\hline Abell1204 & $0.173_{-0.008}^{+0.001}$ & $3.81^{-0.50}+57$ & 0.92 & $0.111_{-0.027}^{+0.009}$ & $0.355 \pm 0.005$ & $1.841 \pm 1.344$ & $0.744 \pm 0.003$ \\
\hline Abell383 & $0.191_{-0.005}^{+0.008}$ & $\begin{array}{l}0.57 \\
5.05_{-0.31}^{+0.26}\end{array}$ & 1.03 & $0.060_{-0.014}^{+0.027}$ & $0.254 \pm 0.003$ & $1.387 \pm 0.297$ & $0.913 \pm 0.002$ \\
\hline RXJ0439.0+0520 & $0.203_{-0.011}^{+0.005}$ & $4.51_{-0.50}^{+0.31}$ & 0.98 & $0.167_{-0.029}^{+0.014}$ & $0.292 \pm 0.005$ & $2.967 \pm 1.519$ & $0.759 \pm 0.002$ \\
\hline ZwCL2701 & $0.214_{-0.004}^{+0.0011}$ & $\begin{array}{r}-0.50 \\
5.65_{-0.44}^{+0.47}\end{array}$ & 1.11 & $0.038^{+0.0023}$ & $0.153 \pm 0.002$ & $1.753 \pm 0.433$ & $0.989 \pm 0.001$ \\
\hline RXJ1504.1-0248 & $0.214_{-0.002}^{+0.004}$ & $8.52^{-0.0 .34}$ & 1.35 & $0.158_{-0.061}^{+0.1017}$ & $0.336 \pm 0.002$ & $3.488 \pm 0.769$ & $2.714 \pm 0.002$ \\
\hline ZwCL2089 & $0.229_{-0.010}^{+0.002}$ & $5.04_{-0.44}^{+0.34}$ & 1.02 & $0.083_{-0.043}^{+0.061}$ & $0.292 \pm 0.004$ & $0.996 \pm 0.390$ & $0.868 \pm 0.002$ \\
\hline RXJ2129.6+0005 & $0.236_{-0.005}^{+0.010}$ & $7.36^{-0.0 .58}$ & 1.24 & $0.048^{+0.0055}$ & $0.188 \pm 0.003$ & $1.336 \pm 0.390$ & $1.662 \pm 0.002$ \\
\hline RXJ1459.4-1811 & $0.233_{-0.004}^{+0.005}$ & $7.27^{+0.1 .32}$ & 1.24 & $0.153^{-0.8014}$ & $0.222 \pm 0.003$ & $6.534 \pm 3.490$ & $1.479 \pm 0.004$ \\
\hline Abell1835 & $0.251_{-0.002}^{+0.004}$ & $9.60^{+1.2 .27}$ & 1.42 & $0.156^{+0.0154}$ & $0.231 \pm 0.001$ & $5.157 \pm 1.887$ & $2.955 \pm 0.001$ \\
\hline Abell3444 & $\begin{array}{l}-0.002 \\
0.258^{+0.005}\end{array}$ & $8.53^{+0.261}$ & 1.33 & $0.051^{+0.0 .109}$ & $0.189 \pm 0.002$ & $1.474 \pm 0.492$ & $2.290 \pm 0.004$ \\
\hline MS1455.0+2232 & $0.259^{+0.0006}$ & $\begin{array}{l}-0.85 \\
5.10^{+0.13}\end{array}$ & 1.02 & $0.093^{-0.0 .032}$ & $0.280 \pm 0.002$ & $1.722 \pm 0.233$ & $1.205 \pm 0.001$ \\
\hline MS2137.3-2353 & $315^{-0.0003}$ & $5.93^{-0.23}$ & 1.06 & $0.051^{-0.00 .027}$ & $0.316 \pm 0.002$ & $0.853 \pm 0.197$ & $1.307 \pm 0.002$ \\
\hline MACSJ2229.7-2755 & $0.329_{-0.000}^{+0.003}$ & $4.38^{-0.25}$ & 0.90 & $0.042_{-0.023}^{+0.028}$ & $0.338 \pm 0.006$ & $0.976 \pm 0.243$ & $0.924 \pm 0.003$ \\
\hline MACSJ0947.2+7623 & $0.353_{-0.006}^{+0.009}$ & $8.19_{-0.71}^{+0.71}$ & 1.24 & $0.100_{-0.044}^{+0.121}$ & $0.280 \pm 0.003$ & $3.740 \pm 2.864$ & $2.138 \pm 0.004$ \\
\hline MACSJ1931.8-2634 & $0.355_{-0.003}^{+0.006}$ & $8.36_{-0.40}^{+0.39}$ & 1.24 & $0.123_{-0.056}^{+0.044}$ & $0.254 \pm 0.002$ & $2.111 \pm 0.364$ & $1.962 \pm 0.002$ \\
\hline MACSJ1115.8+0129 & $0.358_{-0.006}^{+0.005}$ & $8.54_{-0.07}^{+0.50}$ & 1.26 & $0.060_{-0.030}^{+0.056}$ & $0.194 \pm 0.003$ & $3.035 \pm 1.638$ & $2.301 \pm 0.005$ \\
\hline RXJ1532.9- & $0.358_{-0.007}^{+0.006}$ & $6.89_{-0.50}^{+0.50}$ & 1.13 & $0.060_{-0.034}^{+0.030}$ & $0.251 \pm 0.002$ & $1.913 \pm 0.560$ & $2.079 \pm 0.003$ \\
\hline MACSJ0011.7-1523 & $\begin{array}{l}0.376_{-0.009}^{+0.007} \\
0.009\end{array}$ & $6.81_{-0.42}^{+0.59}$ & 1.11 & $0.076_{-0.035}^{+0.034}$ & $0.148 \pm 0.003$ & $1.208 \pm 0.416$ & $1.379 \pm 0.004$ \\
\hline MACSJ1720.2+3536 & $0.389_{-0.005}^{-0.009}$ & $\begin{array}{l}-0.42 \\
6.37_{-0.50}^{+0.55}\end{array}$ & 1.05 & $0.045_{-0.026}^{-0.035}$ & $0.200 \pm 0.004$ & $1.327 \pm 0.571$ & $1.294 \pm 0.003$ \\
\hline MACSJ0429.6-0253 & $0.405_{-0.008}^{+0.005}$ & $5.24_{-0.71}^{+0.710}$ & 0.95 & $0.040_{-0.022}^{+0.026}$ & $0.275 \pm 0.007$ & $1.709 \pm 1.399$ & $1.066 \pm 0.003$ \\
\hline MACSJ0159.8-0849 & $0.408_{-0.007}^{+0.008}$ & $9.62^{+0.79}$ & 1.30 & $0.044_{-0.017}^{+0.022}$ & $0.177 \pm 0.003$ & $3.589 \pm 1.129$ & $2.521 \pm 0.004$ \\
\hline MACSJ2046.0-3430 & $0.424_{-0.008}^{+0.007}$ & $4.71^{-0.52} .47$ & 0.88 & $0.038^{+0.0058}$ & $0.271 \pm 0.006$ & $0.947 \pm 0.320$ & $0.963 \pm 0.003$ \\
\hline IRAS09104+4109 & $0.446_{-0.015}^{+0.008}$ & $\begin{array}{l}-0.48 \\
5.47^{+0.67}\end{array}$ & 0.95 & $0.042^{-0.0051}$ & $0.319 \pm 0.005$ & $0.983 \pm 0.141$ & $1.003 \pm 0.003$ \\
\hline MACSJ0329.6-0211 & $\begin{array}{r}-0.015 \\
55^{+0.002}\end{array}$ & $6.88^{-0.67}+66$ & 1.06 & $0.086_{-0.027}^{+0.052}$ & $0.249 \pm 0.004$ & $2.292 \pm 0.713$ & $1.364 \pm 0.002$ \\
\hline MACSJ1621.3+3810 & $0.464^{+0.0008}$ & $8.60^{-1.034}$ & 1.19 & $0.076^{+0.03076}$ & $0.205 \pm 0.003$ & $1.326 \pm 0.467$ & $1.387 \pm 0.002$ \\
\hline $3 \mathrm{C} 295$ & $0.474^{+0.0005}$ & $\begin{array}{l}-1.08 \\
5.96^{+0.46}\end{array}$ & 1.22 & $0.029^{+0.038}$ & $0.262 \pm 0.005$ & $1.532 \pm 0.813$ & $1.123 \pm 0.003$ \\
\hline MACSJ14 & $45^{-0.8085}$ & $6.32^{-0.461}$ & 0.97 & $0.071^{-0.01072}$ & $0.310 \pm 0.004$ & $1.714 \pm 0.638$ & $1.099 \pm 0.002$ \\
\hline SPT- & $\begin{aligned}-0.004 \\
576^{+0.008}\end{aligned}$ & $8.01^{+1.31}$ & 1.07 & $0.087^{+0.0032}$ & $0.181 \pm 0.005$ & $2.591 \pm 2.428$ & $0.961 \pm 0.003$ \\
\hline SPT-CLJ2344-4242 & $\begin{array}{r}-0.008 \\
0.612^{+0.009}\end{array}$ & $8.90^{+1}$ & 1.11 & $0.042_{-0.020}^{+0.021}$ & $0.334 \pm 0.008$ & $4.691 \pm 4.818$ & $2.693 \pm 0.007$ \\
\hline SPT-CLJ0000-5748 & $0.701_{-0.009}^{+0.011}$ & $6.32_{-0}^{+0}$ & 0.88 & $0.034_{-0.019}^{+0.040}$ & $0.209 \pm 0.006$ & $1.488 \pm 0.434$ & $0.713 \pm 0.002$ \\
\hline SPT-CLJ2043-5035 & $0.715_{-0.008}^{+0.009}$ & $5.73_{-0.50}^{+0.52}$ & 0.83 & $0.036_{-0.020}^{+0.040}$ & $0.237 \pm 0.005$ & $0.898 \pm 0.242$ & $0.766 \pm 0.001$ \\
\hline PLCKG266.6 & $0.942_{-0.008}^{+0.008}$ & $11.64_{-0.90}^{-1.50}$ & 1.06 & $0.035_{-0.020}^{+0.020}$ & $0.098 \pm 0.002$ & $2.415 \pm 1.295$ & $1.922 \pm 0.003$ \\
\hline CLJ1415+3612 & $1.036_{-0.013}^{+0.013}$ & $\begin{array}{l}6.40_{-0.65}^{+0.69} \\
6.65\end{array}$ & 0.73 & $0.028_{-0.015}^{+0.035}$ & $0.137 \pm 0.004$ & $1.021 \pm 0.387$ & $0.433 \pm 0.001$ \\
\hline
\end{tabular}

tribution of iron in the ICM. Since we only investigate the most relaxed clusters in our sample, the broadening of the iron peak should not be associated with large scale motions of the ICM, such as those due to major mergers, but it may be associated with the turbulent mixing and uplifting due to the feedback activities of the central galaxy. Another mechanism that could contribute to the widening of the peak is the core sloshing due to minor mergers (see the discussion of A496 in Ghizzardi et al. 2014).

Another important clue comes from the mass of the iron excess, computed within $0.3 r_{500}$. In Figure 9, we plot the iron mass excess normalized by the gas mass within $0.3 r_{500}$ versus redshift. Spearman's test on the distribution gives $\rho=-0.09 \pm 0.12$, and the probability of no evolution $p=$
$0.50 \pm 0.29$, suggesting no significant evolution of iron mass excess with redshift. Therefore, we conclude that the bulk of the mass in iron is already present at $z \sim 1$, and that the increase in the quantity $r_{\mathrm{Fe}}$ should not be ascribed to an increase of the iron excess in the ICM with cosmic epoch, but mostly to the evolution of the iron distribution.

\section{CONCLUSIONS}

We perform a systematic study on the evolution of iron distribution in the ICM with deprojected $Z_{\mathrm{Fe}}$ profiles in a sample of 41 relaxed galaxy clusters in the redshift range $0.05<z<1.03$. Our conclusions are summarized as follows: 


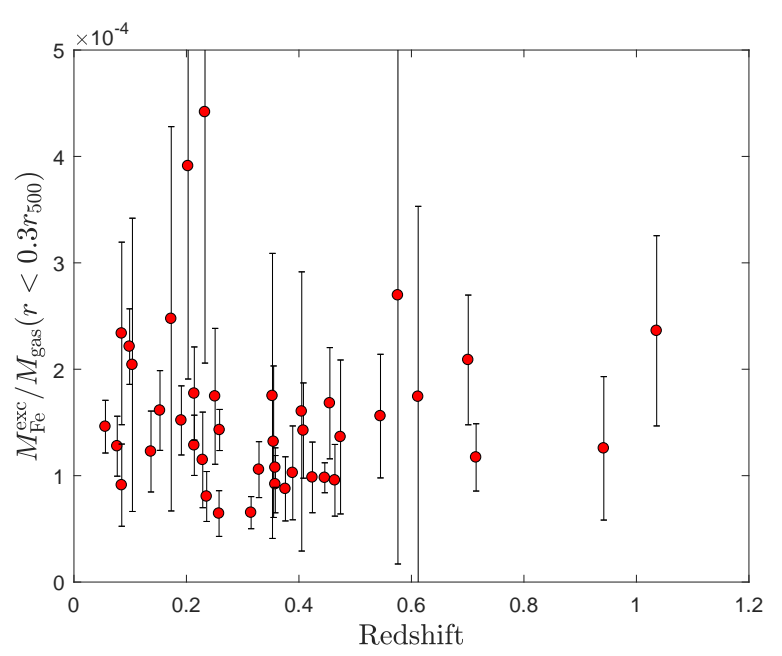

Figure 9. The mass of the Fe excess, normalized to the total gas mass, within $0.3 r_{500}$ as a function of redshift.

- We confirm that for all of our clusters from $z \sim 0.05$ to $z \sim 1.03$, the shape of the deprojected $Z_{\mathrm{Fe}}$ radial profiles shows a steep negative gradient followed by a roughly constant value out to $\sim 0.4 r_{500}$, as commonly observed in relaxed/cool-core clusters. The average $Z_{\mathrm{Fe}}$ at $\sim 0.3 r_{500}$ is approximately $0.3 Z_{\odot}$, and shows no significant evolution with redshift.

- With the deprojected iron profile, we calculate the mass weighted iron abundance $Z_{\mathrm{mw}}$ within $0.2 r_{500}$ for the clusters, and make a comparison with the average iron abundance $\left\langle Z_{\mathrm{Fe}}\right\rangle$ which is obtained by simply fitting the overall emission within the same radius. As a result, we find that the average value is always larger than the mass weighted value by $\sim 25 \%$, showing a potential issue when computing the cosmic evolution of the global enrichment of the ICM without having its spatial distribution under control.

- We investigate the correlation between $Z_{\mathrm{Fe}}$ and the pseudo entropy $K_{T, n_{e}}$, and the correlation between $Z_{\mathrm{Fe}}$ and the cooling time $t_{\text {cool }}$ in all the measured bins of all clusters. We confirm that higher $Z_{\mathrm{Fe}}$ corresponds to lower $K\left(k T, n_{e}\right)$ and shorter $t_{\mathrm{cool}}$, with large scatters. We suggest that this association is relevant to the interplay of the radiative cooling of the gas and the production and diffusion of iron.

- We quantify the width of the iron profiles with the parameter $r_{\mathrm{Fe}}$, defined as the radius where the iron abundance excess is half of its maximum value. We find that $r_{\mathrm{Fe}}$ decreases significantly with redshift, but not with the core strength, and that the total mass excess in iron is not evolving with redshift. This shows that we are witnessing the evolution in the distribution of the iron mass excess in the inner region of cool cores, possibly associated with the turbulent mixing and uplifting of highly enriched material due to the mechanical-mode feedback from the central galaxy.

This work is the first on a series of papers aiming at establishing a robust modeling of the iron distribution in the ICM based on a central peak and a large scale flat component. This can be obtained thanks to a detailed analysis of bright, low and moderate $z$ clusters, as in this paper. Eventually, the evolution of the iron abundance across the largest cluster population observable to date in terms of mass and redshift range, can be investigated by using these spatial distribution models.

\section{ACKNOWLEDGEMENTS}

We thank the anonymous referee for his/her helpful comments. We acknowledge financial contribution from the agreement ASI-INAF n.2017-14-H.O. S.E. acknowledges also financial contribution from the contracts NARO15 ASIINAF I/037/12/0, and ASI 2015-046-R.0.

\section{REFERENCES}

Allen S. W., Evrard A. E., Mantz A. B., 2011, ARA\&A, 49, 409 Anderson M. E., Bregman J. N., Butler S. C., Mullis C. R., 2009, ApJ, 698, 317

Arnaud K. A., 1996, in Jacoby G. H., Barnes J., eds, Astronomical Society of the Pacific Conference Series Vol. 101, Astronomical Data Analysis Software and Systems V. p. 17

Asplund M., Grevesse N., Sauval A. J., Scott P., 2009, ARA\&A, 47,481

Baldi A., Ettori S., Molendi S., Balestra I., Gastaldello F., Tozzi P., 2012, A\&A, 537, A142

Balestra I., Tozzi P., Ettori S., Rosati P., Borgani S., Mainieri V., Norman C., Viola M., 2007, A\&A, 462, 429

Bartalucci I., Arnaud M., Pratt G. W., Démoclès J., van der Burg R. F. J., Mazzotta P., 2017, A\&A, 598, A61

Boehringer H., Hensler G., 1989, A\&A, 215, 147

Böhringer H., Werner N., 2010, A\&ARv, 18, 127

Böhringer H., Matsushita K., Churazov E., Finoguenov A., Ikebe Y., 2004, A\&A, 416, L21

Cash W., 1979, ApJ, 228, 939

Cora S. A., 2006, MNRAS, 368, 1540

Cora S. A., Tornatore L., Tozzi P., Dolag K., 2008, MNRAS, 386, 96

De Grandi S., Molendi S., 2001, ApJ, 551, 153

De Grandi S., Molendi S., 2009, A\&A, 508, 565

De Grandi S., Ettori S., Longhetti M., Molendi S., 2004, A\&A, 419,7

De Grandi S., Santos J. S., Nonino M., Molendi S., Tozzi P., Rossetti M., Fritz A., Rosati P., 2014, A\&A, 567, A102

Ettori S., Baldi A., Balestra I., Gastaldello F., Molendi S., Tozzi P., 2015, A\&A, 578, A46

Fabian A. C., 2012, ARA\&A, 50, 455

Fabian A. C., Sanders J. S., Taylor G. B., Allen S. W., Crawford C. S., Johnstone R. M., Iwasawa K., 2006, MNRAS, 366, 417

Finoguenov A., David L. P., Ponman T. J., 2000, ApJ, 544, 188

Ghizzardi S., De Grandi S., Molendi S., 2014, A\&A, 570, A117

Hitomi Collaboration et al., 2017, preprint, (arXiv:1710.04648)

Hudson D. S., Mittal R., Reiprich T. H., Nulsen P. E. J., Andernach H., Sarazin C. L., 2010, A\&A, 513, A37

Kalberla P. M. W., Burton W. B., Hartmann D., Arnal E. M., Bajaja E., Morras R., Pöppel W. G. L., 2005, A\&A, 440, 775

Komatsu E., et al., 2011, ApJS, 192, 18

Kravtsov A. V., Borgani S., 2012, ARA\&A, 50, 353

Leccardi A., Rossetti M., Molendi S., 2010, A\&A, 510, A82

Lin Y.-T., Stanford S. A., Eisenhardt P. R. M., Vikhlinin A., Maughan B. J., Kravtsov A., 2012, ApJ, 745, L3

Madau P., Dickinson M., 2014, ARA\&A, 52, 415

Mantz A. B., Allen S. W., Morris R. G., Schmidt R. W., von der Linden A., Urban O., 2015, MNRAS, 449, 199

Mantz A. B., Allen S. W., Morris R. G., 2016, MNRAS, 462, 681

Mantz A. B., Allen S. W., Morris R. G., Simionescu A., Urban O., Werner N., Zhuravleva I., 2017, MNRAS, 472, 2877 
Maoz D., Graur O., 2017, ArXiv:1703.04540,

Markevitch M., Vikhlinin A., 2007, PhR, 443, 1

Matsushita K., Sakuma E., Sasaki T., Sato K., Simionescu A., 2013, ApJ, 764, 147

Maughan B. J., Jones C., Forman W., Van Speybroeck L., 2008, ApJS, 174, 117

McDonald M., et al., 2016, ApJ, 826, 124

Mernier F., et al., 2017, A\&A, 603, A80

Peterson J. R., Fabian A. C., 2006, PhR, 427, 1

Ponman T. J., Cannon D. B., Navarro J. F., 1999, Nature, 397, 135

Rebusco P., Churazov E., Böhringer H., Forman W., 2005, MNRAS, 359, 1041

Roediger E., Brüggen M., Rebusco P., Böhringer H., Churazov E., 2007, MNRAS, 375, 15

Romeo A. D., Sommer-Larsen J., Portinari L., Antonuccio-Delogu V., 2006, MNRAS, 371, 548

Rosati P., et al., 2009, A\&A, 508, 583

Rossetti M., Gastaldello F., Eckert D., Della Torre M., Pantiri G., Cazzoletti P., Molendi S., 2017, MNRAS, 468, 1917

Russell H. R., Sanders J. S., Fabian A. C., 2008, MNRAS, 390, 1207

Sanders J. S., Fabian A. C., 2007, MNRAS, 381, 1381

Sanders J. S., Fabian A. C., 2011, MNRAS, 412, L35

Santos J. S., Rosati P., Tozzi P., Böhringer H., Ettori S., Bignamini A., 2008, A\&A, 483, 35

Santos J. S., Tozzi P., Rosati P., Nonino M., Giovannini G., 2012, A\&A, 539, A105

Sijacki D., Springel V., Di Matteo T., Hernquist L., 2007, MNRAS, 380, 877

Smith R. K., Brickhouse N. S., Liedahl D. A., Raymond J. C., 2001, ApJ, 556, L91

Sutherland R. S., Dopita M. A., 1993, ApJS, 88, 253

Tamura T., et al., 2009, ApJL, 705, L62

Tornatore L., Borgani S., Matteucci F., Recchi S., Tozzi P., 2004, MNRAS, 349, L19

Tozzi P., Norman C., 2001, ApJ, 546, 63

Tozzi P., Santos J. S., Nonino M., Rosati P., Borgani S., Sartoris B., Altieri B., Sanchez-Portal M., 2013, A\&A, 551, A45

Tozzi P., et al., 2015, ApJ, 799, 93

Vikhlinin A., 2006, ApJ, 640, 710

Vikhlinin A., Kravtsov A., Forman W., Jones C., Markevitch M., Murray S. S., Van Speybroeck L., 2006, ApJ, 640, 691

Werner N., Durret F., Ohashi T., Schindler S., Wiersma R. P. C., 2008, SSRv, 134, 337

Wilms J., Allen A., McCray R., 2000, ApJ, 542, 914

Yu H., Tozzi P., Borgani S., Rosati P., Zhu Z.-H., 2011, A\&A, 529, A65

\section{APPENDIX A: THE DEPROJECTED $Z_{\mathrm{FE}}$ PROFILES OF THE CLUSTERS IN OUR \\ SAMPLE.}



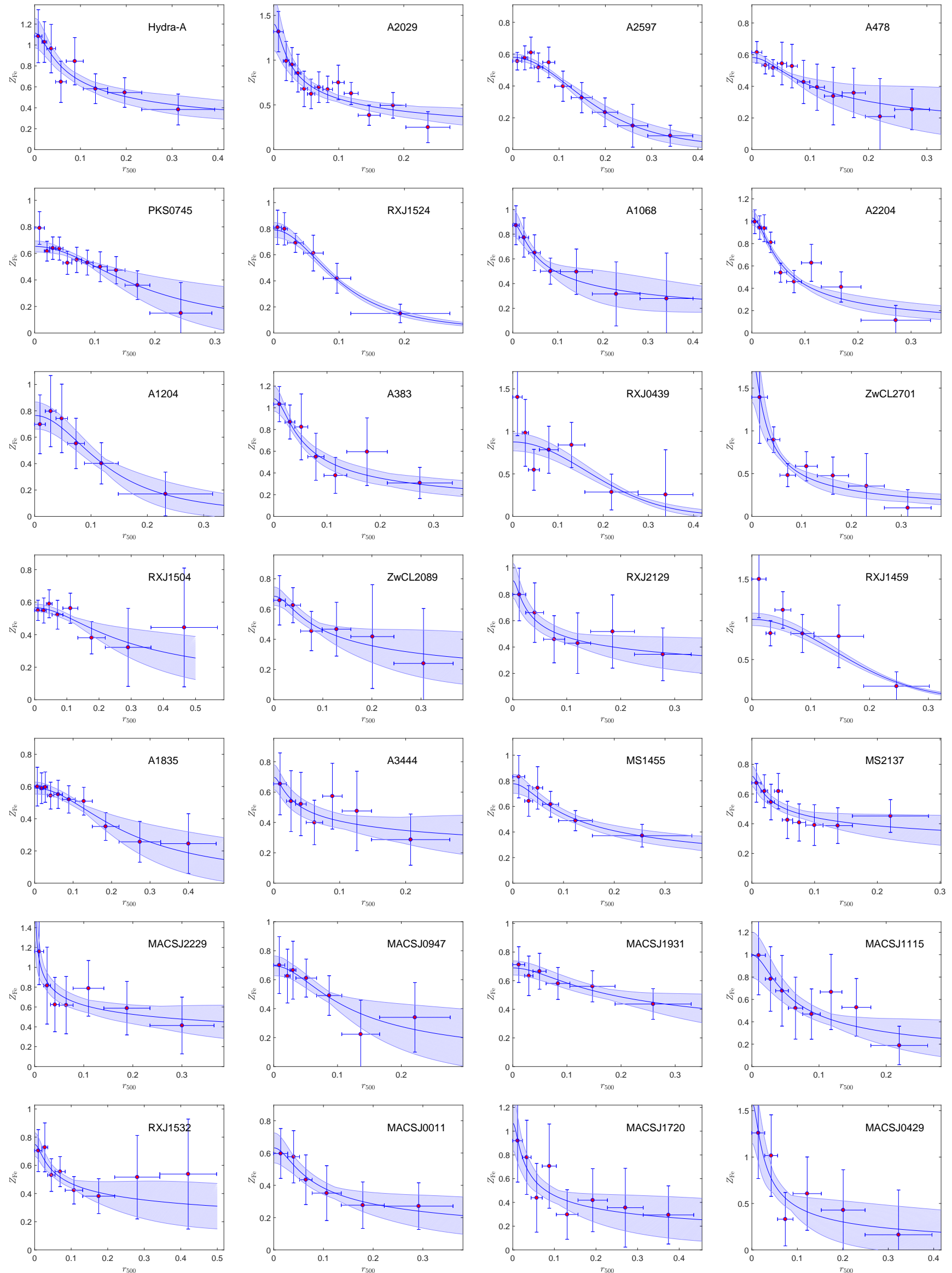

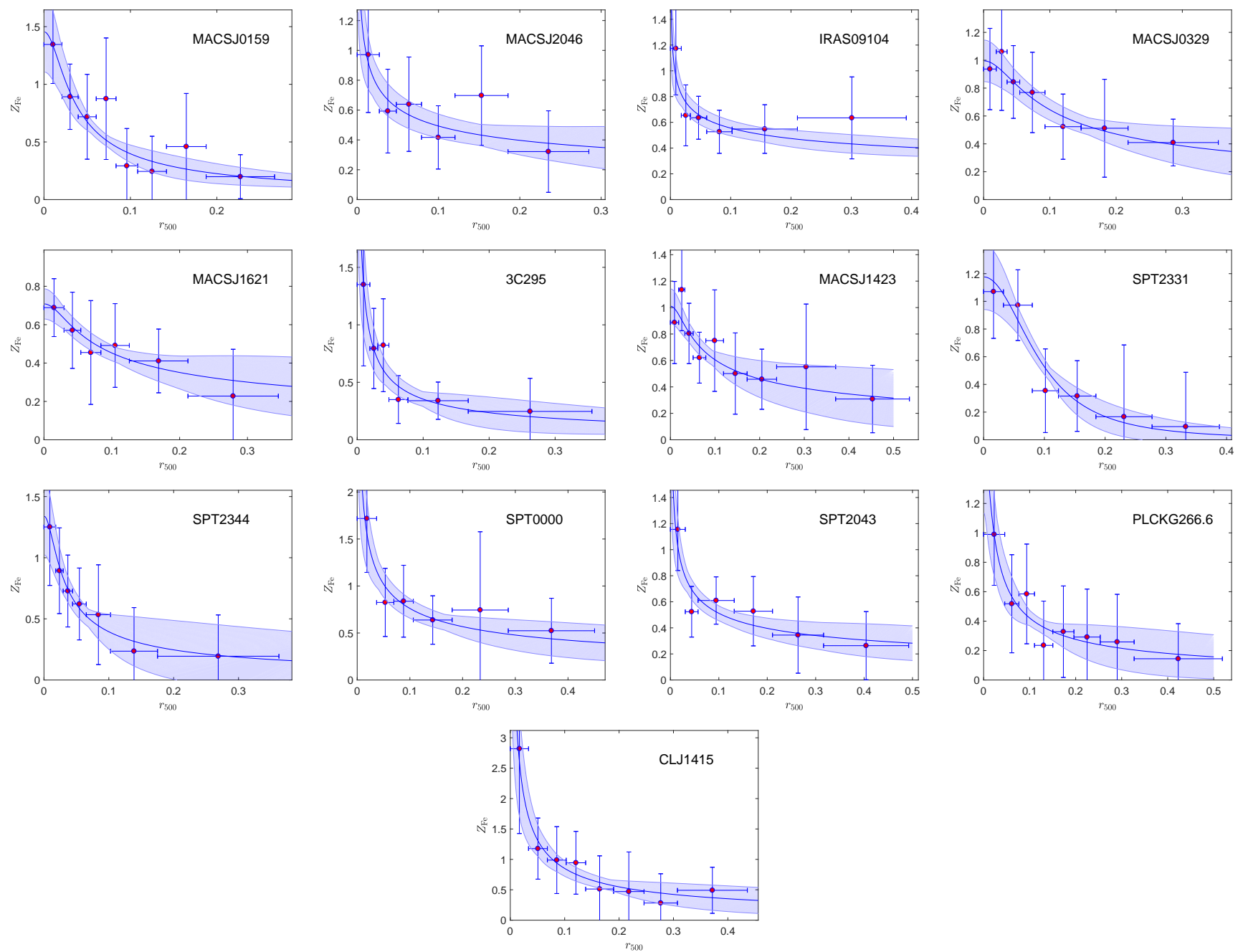

Figure A1. Deprojected radial profiles of iron abundance of the clusters in our sample. The solid blue line shows the best fit model. The shaded area corresponds to $1 \sigma$ confidence interval. 